\title{
Modelado Numérico del Proceso de Pultrusión en Materiales Compuestos
}

\author{
Diego Santiago, Guillermo Lombera*,Santiago Urquiza, Stella M. Moschiar \\ Facultad de Ingeniería, Univ. Nac. de Mar del Plata, \\ J.B. Justo 4302, 7600 Mar del Plata - Bs.As., Argentina
}

Received: February 14, 2003; Revised: June 26, 2003

\begin{abstract}
A mathematical pultrusion model of a thermosetting matrix composite is presented. The energy balance was solved by Finite Elements techniques using a program developed for this purpose. This model is applied to describe the temperature and resin conversion profiles along the die. This study was developed considering different pulling velocity, die temperature, different composite and different sections die. The model outcomes are verified through comparison with the experimental results reported in the literature.
\end{abstract}

Palabras-Clave: materiales compuestos, pultrusión, elementos finitos

\section{Introducción}

El proceso de pultrusión de un termorrígido, se esquematiza en la Fig. 1 y consiste en el mojado de fibras en un baño que contiene la resina y el posterior tirado de las fibras a través de un molde calefaccionado. Cuando la resina pasa por el molde, se va calentando hasta que posee la temperatura a la cual se activa la reacción química. En este momento comienza la reacción de entrecruzamiento o proceso de curado, mediante el cual pasa de ser una resina líquida a un gel. A fin de modelar este proceso se requiere del planteo de un modelo de transferencia de calor, con un submodelo cinético y uno de propiedades térmicas del compuesto.

En los últimos años se han publicado varios trabajos que presentan modelos matemáticos para describir el proceso de pultrusión. Moschiar et al. ${ }^{1,2}$ presentan un análisis de cómo afectan las variables del proceso sobre el material compuesto obtenido en una pultrusión. Los trabajos desarrollan un modelo matemático resuelto por diferencias finitas, utilizando una resina poliéster y una resina epoxi como matrices. La descripción del proceso incluye el modelo para transferencia de calor, curado de la resina, presión desarrollada en el interior del molde, fuerza de tirado y en el segundo trabajo, además la predicción de las fuerzas de tirado.

Sumerak ${ }^{3}$ utiliza un programa de análisis de elementos finitos comercial para resolver un modelo de transferencia de calor en tres dimensiones, en un proceso de pultrusión, teniendo en cuenta la variación del perfil de temperatura dentro del molde. Aplica el modelo a diferentes perfiles con distintos grados de complejidad.

Joshi and $\mathrm{Lam}^{4}$ discuten el desarrollo y aplicación de una aproximación utilizando elementos finitos y volúmenes de control, en tres dimensiones, sobre un perfil irregular obtenido en un proceso de pultrusión. Utiliza un programa comercial de elementos finitos (LUSAS 13.0) para la transferencia de calor por conducción. La transferencia de calor entre el molde y el compuesto que se mueve en él, la modelan usando superficies térmicas, mediante un esquema semi-numérico resuelto con códigos FORTRAN.

El objetivo de este trabajo es el modelado numérico de la transferencia de calor con sus respectivos submodelos para el caso de una resina epoxi y una resina poliéster y su verificación con datos experimentales obtenidos de un pultrusor, utilizando un programa de elementos finitos desarrollado en nuestra facultad implementado en un entorno genérico para la solución de problemas reducibles por elementos. Se pretende además realizar un estudio de las variables de proceso tales como velocidad de tirado y temperatura en la pared, a fin de encontrar las condiciones mejores de procesamiento en cuanto a la transferencia de calor.

*e-mail: glombera@ fi.mdp.edu.ar 
El presente trabajo es la continuación de un proyecto de investigación en el tema de pultrusión, donde se prevé seguir estudiando las alternativas más eficientes para la simulación numérica del proceso, incluyendo estudios termomecánicos a fin de poder predecir las tensiones residuales involucradas.

\section{Modelo Matemático de Transferencia de Calor}

La ecuación general de balance de calor que describe al problema es:

$$
\frac{\mathrm{DT}}{\mathrm{Dt}}=\nabla^{2} \mathrm{~T}+\rho\left(-\Delta \mathrm{H}_{0}\right) \omega_{\mathrm{r}} R_{a} \cdot(1)
$$

Donde $\rho$ es la densidad del compuesto, $\Delta \mathrm{H}_{0}$ es el calor de reacción del polímero, $\omega_{\mathrm{r}}$ es la fracción másica de resina y $R_{\mathrm{a}}=\mathrm{dX} / \mathrm{dt}$ (X grado de avance de la reacción de curado o conversión) es la velocidad de reacción que es distinta para cada resina.

Se asume que el compuesto permanece siempre en contacto con la pared y que la conducción de calor en la dirección axial es despreciable. Para la matriz epoxi se utilizó una simetría de revolución, mientras que para la matriz poliéster una simetría plana. Con esas suposiciones la ecuación de balance de energía a resolver para el caso plano y para el caso de simetría de revolución presenta la forma dada por las Ecs. 2 y 3 respectivamente.

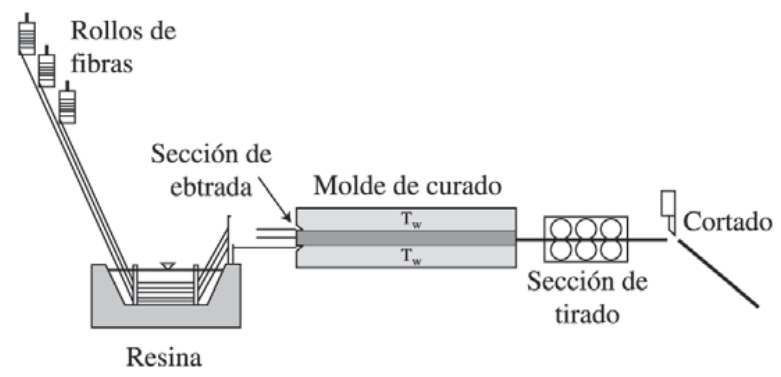

Figura 1. Esquema del proceso de pultrusión.

$$
\begin{aligned}
& \rho \mathrm{C}_{\mathrm{p}}\left(\frac{\partial \mathrm{T}}{\partial \mathrm{t}}+\mathrm{V}_{\mathrm{z}} \frac{\partial \mathrm{T}}{\partial \mathrm{z}}\right)=\mathrm{k}\left(\frac{\partial^{2} \mathrm{~T}}{\partial \mathrm{x}^{2}}+\frac{\partial^{2} \mathrm{~T}}{\partial \mathrm{z}^{2}}\right)+\rho\left(-\Delta \mathrm{H}_{0}\right) \omega_{\mathrm{r}} \mathrm{R}_{\mathrm{a}} \\
& \rho \mathrm{C}_{\mathrm{p}}\left(\frac{\partial \mathrm{T}}{\partial \mathrm{t}}+\mathrm{V}_{\mathrm{z}} \frac{\partial \mathrm{T}}{\partial \mathrm{z}}\right)=\frac{\mathrm{k}}{\mathrm{r}} \frac{\partial}{\partial \mathrm{r}}\left(\mathrm{r} \frac{\partial \mathrm{T}}{\partial \mathrm{r}}\right)+\mathrm{k} \frac{\partial^{2} \mathrm{~T}}{\partial \mathrm{z}^{2}}+\rho\left(-\Delta \mathrm{H}_{0}\right) \omega_{\mathrm{r}} \mathrm{R}_{\mathrm{a}}
\end{aligned}
$$

Donde $\mathrm{C}_{\mathrm{p}}$ es la capacidad calorífica del compuesto, $\mathrm{V}_{\mathrm{Z}}$ es la velocidad de tirado del pultrusor y $\mathrm{k}$ es la conductividad térmica del compuesto en la dirección $\mathrm{x}$.

Las Ecs. 2 y 3 son complementadas con adecuadas condiciones de contorno que de acuerdo a las configuraciones geométricas resultan:

Para el caso de la geometría plana ( resina poliéster):

$$
\begin{aligned}
& 0 \leq \mathrm{x} \leq \mathrm{b} \quad \mathrm{z}=0 \quad \mathrm{~T}=\mathrm{T}_{0} \quad \mathrm{x}=0 \quad \mathrm{I}_{0 \mathrm{i}}=0 \\
& \mathrm{x}=\mathrm{b} \quad 0 \leq \mathrm{z} \leq \mathrm{L} \quad \mathrm{T}=\mathrm{T}_{\mathrm{w}}=\mathrm{T}_{\mathrm{w}}(\mathrm{z}) \\
& \mathrm{x}=0 \quad 0 \leq \mathrm{z} \leq \mathrm{L} \quad \partial \mathrm{T} / \partial \mathrm{x}=0
\end{aligned}
$$

donde $b$ es el ancho del perfil plano y L es el largo del molde del pultrusor.

Para el caso de simetría de revolución (resina epoxi):

$$
\begin{array}{ccc}
0 \leq \mathrm{r} \leq \mathrm{R} & \mathrm{z}=0 \quad \mathrm{~T}=\mathrm{T}_{0} \quad \mathrm{x}=0 \\
\mathrm{r}=\mathrm{R} & 0 \leq \mathrm{z} \leq \mathrm{L} \quad \mathrm{T}=\mathrm{T}_{\mathrm{w}}=\mathrm{T}_{\mathrm{w}}(\mathrm{z}) \\
\mathrm{r}=\mathrm{0} & 0 \leq \mathrm{z} \leq \mathrm{L} \quad \mathrm{\partial T} / \mathrm{\partial r}=0
\end{array}
$$

donde $\mathrm{R}$ es el radio del cilindro y L es el largo del molde del pultrusor.

Los modelos cinéticos para cada resina son los que aparecen en los trabajos de Moschiar et al. ${ }^{1,2}$.

El sistema seleccionado en el primer caso fue una resina poliéster isoftálica (Kopper Dion 8200) con una mezcla de iniciadores $\left(\mathrm{I}_{\mathrm{i}}\right)$ que consisten en Percadox 16 (PeroxidicarbonatoP16N), t-butil peroctato (TBPO) y t-butilperbenzoato (TBPB). La expresión cinética utilizada fue la siguiente:

Tabla 1. Datos cinéticos de los iniciadores.

\begin{tabular}{ccc}
\hline Concentración & Factor pre-exponencial & Energía de activación \\
\hline $\mathrm{I}_{01}=53,7$ & $\mathrm{Ad}_{1}=2,41 \times 10^{17}$ & $\mathrm{Ed}_{1} / \mathrm{R}=16250$ \\
$I_{02}=46,3$ & $\mathrm{Ad}_{2}=2,92 \times 10^{16}$ & $\mathrm{Ed}_{2} / \mathrm{R}=17100$ \\
$I_{03}=12,9$ & $\mathrm{Ad}_{3}=7,76 \times 10^{13}$ & $\mathrm{Ed}_{3} / \mathrm{R}=15060$ \\
\hline
\end{tabular}




$$
\begin{gathered}
R_{\mathrm{a}}=\frac{\mathrm{dX}}{\mathrm{dt}}=3.244 \times 10^{5} \exp \left(-\frac{55.10}{\mathrm{R} \mathrm{T}}\right) \\
\cdot\left(\mathrm{I}_{0}-\mathrm{I}\right) \cdot(1-\mathrm{X}) \cdot\left(1-\frac{\mathrm{X}}{0.9}\right)\left[\mathrm{s}^{-1}\right] \\
\frac{\mathrm{dI}_{\mathrm{i}}}{\mathrm{dt}}=-A d_{i} \exp \left(-\frac{\mathrm{Ed}}{\mathrm{RT}}\right) \cdot \mathrm{I}_{\mathrm{i}} \quad \mathrm{I}=\sum \mathrm{I}_{\mathrm{i}}
\end{gathered}
$$

donde los datos de los iniciadores $\left(\mathrm{I}_{\mathrm{i}}\right)$, es decir las concentraciones, energías de activación y factores preexponenciales de cada uno se dan en la Tabla 1. Los iniciadores, son sustancias que permiten que la polimerización de la resina poliéster comience. En este caso se necesitó una mezcla de tres sustancias, para obtener una óptima velocidad de reacción.

El segundo sistema estudiado fue una resina epoxi Epon 9310 usando un agente de curado comercial 9360 y Epon 537 como acelerador. La expresión cinética fue la siguiente:

$$
R_{\mathrm{a}}=\frac{\mathrm{dX}}{\mathrm{dt}}=2.918 \times 10^{6} \exp \left(-\frac{66.013}{\mathrm{RT}}\right) \cdot(1-\mathrm{X})^{2}\left[s^{-1}\right]
$$

Si bien se intenta resolver el caso estacionario, el término temporal no se despreció, ya que facilita alcanzar la solución del problema en forma iterativa.

Las ecuaciones anteriores referidas a las conversiones $\mathrm{X}$ y los indicadores I - que representaremos aquí genéricamente para ambos casos por $\mathrm{C}_{\mathrm{i}}$ - están referidas a una masa definida de reactivo, por lo tanto deben ser implementadas como ecuaciones de advección-reacción rescribiendo la derivada temporal como derivada material o lagrangiana:

$$
\frac{\mathrm{dC}_{\mathrm{i}}}{\mathrm{dt}} \rightarrow \frac{\partial \mathrm{C}_{\mathrm{i}}}{\partial \mathrm{t}}+\mathrm{V}_{\mathrm{z}} \frac{\partial \mathrm{C}_{\mathrm{i}}}{\partial \mathrm{z}}
$$

Por lo tanto, las Ecs. 6, 7 y 8 pueden ser escritas de manera genérica como

$$
\frac{\partial \mathrm{C}_{\mathrm{i}}}{\partial \mathrm{t}}+\mathrm{V}_{\mathrm{z}} \frac{\partial \mathrm{C}_{\mathrm{i}}}{\partial \mathrm{z}}=R_{i}\left(C_{j}, T\right)
$$

donde $\mathrm{R}_{\mathrm{i}}$ representan los diferentes términos de reacción (iguales a los miembros del lado derecho del igual de las ecuaciones referidas anteriormente).

\section{Propiedades Físicas del Compuesto}

En el presente trabajo se utilizaron un compuesto de matriz epoxi y otro cuya matriz es una resina poliéster insaturada. Las propiedades físicas se calcularon utilizando la regla de mezclas según las siguientes ecuaciones.

$$
\begin{aligned}
& \rho=\phi_{\mathrm{r}} \rho_{\mathrm{r}}+\phi_{\mathrm{g}} \rho_{\mathrm{g}}+\phi_{\mathrm{f}} \rho_{\mathrm{f}} \\
& \mathrm{C}_{\mathrm{p}}=\omega_{\mathrm{r}} \mathrm{C}_{\mathrm{pr}}+\omega_{\mathrm{g}} \mathrm{C}_{\mathrm{pg}}+\omega_{\mathrm{f}} \mathrm{C}_{\mathrm{pf}} \\
& \frac{1}{\mathrm{k}}=\frac{\phi_{\mathrm{r}}}{\mathrm{k}_{\mathrm{r}}}+\frac{\phi_{\mathrm{g}}}{\mathrm{k}_{\mathrm{g}}}+\frac{\phi_{\mathrm{f}}}{\mathrm{k}_{\mathrm{f}}}
\end{aligned}
$$

Donde $\omega$ es la fracción másica, $\phi$ la fracción volumétrica y los subíndices representan: r: resina, g: fibras y f: relleno. En la Tabla 2 se muestran los valores de las propiedades físicas de las resinas, las fibras y los rellenos utilizados para calcular las propiedades del compuesto.

Tabla 2. Propiedades físicas.

\begin{tabular}{ccc}
\hline & Poliéster & Epoxi \\
\hline$\rho_{\mathrm{r}}\left(\mathrm{Kg} / \mathrm{m}^{3}\right)$ & 1100 & $-0,1 \cdot \mathrm{T}\left({ }^{\circ} \mathrm{C}\right)+90 \cdot \mathrm{X}+1234($ para X<0,45 $)$ \\
$\mathrm{Cp}$ & & $-0,1 \cdot \mathrm{T}\left({ }^{\circ} \mathrm{C}\right)+1234 \quad(\mathrm{para} \mathrm{X}>0,45)$ \\
$\mathrm{K}_{\mathrm{r}}(\mathrm{KJ} / \mathrm{Kg} \cdot \mathrm{K})$ & $1,883)$ & $1,958+2,5 \cdot 10^{-3} \cdot \mathrm{T}\left({ }^{\circ} \mathrm{C}\right)-0,59 \cdot \mathrm{X}$ \\
$\rho_{\mathrm{g}}\left(\mathrm{Kg} / \mathrm{m}^{3}\right)$ & 0,169 & $0,161+0,04184 \cdot\left(0,035 \cdot \mathrm{T}\left({ }^{\circ} \mathrm{C}\right)-0,41\right)$ \\
$\mathrm{C} \mathrm{p}_{\mathrm{g}}(\mathrm{KJ} / \mathrm{Kg} \cdot \mathrm{K})$ & 2540 & 2540 \\
$\mathrm{k}_{\mathrm{g}}(\mathrm{J} / \mathrm{m} . \mathrm{s})$ & 0,833 & 0,833 \\
$\rho_{\mathrm{f}}\left(\mathrm{Kg} / \mathrm{m}^{3}\right)$ & 0,866 & 0,866 \\
$\mathrm{C} \mathrm{p}_{\mathrm{f}}(\mathrm{KJ} / \mathrm{Kg} \cdot \mathrm{K})$ & 2580 & - \\
$\mathrm{k}_{\mathrm{f}}(\mathrm{J} / \mathrm{m} \cdot \mathrm{s})$ & 0,920 & - \\
\hline
\end{tabular}

(T: Temperatura; X: Conversión ) 


\section{Modelo Numérico}

Para modelar numéricamente el proceso, se utilizó un programa en elementos finitos desarrollado en un entorno de trabajo genérico para la solución de problemas reducibles por elementos. Dicho entorno es aplicable a la resolución de sistemas de ecuaciones diferenciales en derivadas parciales por técnicas de Elementos Finitos, Diferencias Finitas, Volúmenes Finitos, etc., y en general, a todos aquellos problemas que se resuelven a través de sistemas de ecuaciones algebraicas, cuya matriz global pueda construirse por intermedio de contribuciones aditivas o no-aditivas realizadas por "elementos"

El programa esta basado en el concepto de "Elemento", entendido como un conjunto de nodos que tienen asociados un cierto numero de incógnitas o grados de libertad y determinadas relaciones de acoplamiento entre ellas, definidas localmente, i.e., independientemente de otros elementos. Cada nodo puede presentar un numero distinto de grados de libertad, ensamblándose sólo las ecuaciones correspondientes a los grados de libertad efectivamente presentes. Las condiciones de borde pueden ser tratadas de manera convencional o como elementos especiales de contorno. La tarea fundamental del usuario consiste en configurar los datos de entrada, como por ejemplo la descripción de las mallas de nodos y elementos, y las propiedades u otras constantes físicas necesarias, y de programar la matriz elemental si esta no formase parte de la biblioteca existente. El programa basa su generalidad y versatilidad en un ensamblador simbólico y numérico de carácter general, independiente de la clase de elementos a ensamblar, el usuario debe proveer una matriz que refleje la estructura de acoplamientos entre los diferentes grados de libertad del elemento, tarea relativamente sencilla a partir de los bloques de código que se usan para calcular la matriz numérica elemental.

El sistema de ecuaciones local, es decir, para un elemento no acoplado, expresado en forma matricial es:

$$
\left[\mathrm{K}_{\mathrm{lm}}\right] *\left[\alpha_{\mathrm{m}}\right]=\left[\mathrm{B}_{1}\right]
$$

donde $\mathrm{K}_{\mathrm{lm}}$ es la matriz de masas, $\alpha_{\mathrm{m}}$ es el vector de incógnitas del sistema y $\mathrm{B}_{1}$ es el vector de términos independientes. Los subíndices $l$ y $m$ dependen de la cantidad de grados de libertad del sistema, del orden en que se ingresaron los grados de libertad y del número de nodos del elemento. Las dimensiones de la matriz $\mathrm{K}_{\mathrm{lm}}$ y de los vectores $\alpha_{\mathrm{m}}$ y $\mathrm{B}_{1}$ están determinadas por el número de grados de libertad que tiene el sistema y el número de nodos del elemento.

Los subíndices 1 y $m$ están definidos por las siguientes expresiones:

$$
1=\operatorname{DofT} *(i-1)+\mathrm{N}^{\circ} \operatorname{Dof}
$$

Tabla 3. Ingreso de los grados de libertad.

\begin{tabular}{ccc}
\hline NoDof & Variable & $\begin{array}{c}\text { Notación } \\
\text { abreviada }\end{array}$ \\
\hline 1 & Temperatura & $\mathrm{T}$ \\
2 & Conversión de curado & $\mathrm{X}$ \\
3 & Concentración del iniciador $\mathrm{N}^{\circ} 1$ & $\mathrm{I} 1$ \\
4 & Concentración del iniciador $\mathrm{N}^{\circ} 2$ & $\mathrm{I} 2$ \\
5 & Concentración del iniciador $\mathrm{N}^{\circ} 3$ & $\mathrm{I} 3$ \\
\hline
\end{tabular}

$$
m=\operatorname{DofT} *(j-1)+\mathrm{N}^{\circ} \operatorname{Dof}
$$

donde DofT es el número total de grados de libertad que tiene el sistema, $\mathrm{N}^{\circ}$ Dof es el orden en que se ingresó el grado de libertad en el vector de incógnitas (primero $\Rightarrow \mathrm{N}^{\circ}$ Dof $=1$, segundo $\Rightarrow \mathrm{N}^{\circ}$ Dof $=2, \ldots$. , último $\Rightarrow \mathrm{N}^{\circ}$ Dof $=$ DofT $), j$ es el subíndice que se utiliza para denominar las funciones de forma nodales, $\phi_{\mathrm{j}}\left(1,2, . ., \mathrm{N}^{\mathrm{o}}\right.$ de nodos totales del elemento) e $i$ es el subíndice que se utiliza para denominar las funciones de peso nodales, $\varphi_{i}\left(1,2, . ., \mathrm{N}^{\circ}\right.$ de nodos totales del elemento).

Para facilitar la comprensión del proceso de ensamblaje de la matriz de masas y el vector de términos independientes se describirá dicho proceso para el caso del compuesto Poliéster-Fibra de vidrio.

En este caso es necesario resolver el problema para cinco grados de libertad, es decir, DofT $=5$. Los grados de libertad se ingresaron como muestra la Tabla 3.

Por lo tanto, para un elemento triangular de tres nodos $\left(\mathrm{N}^{\mathrm{o}}\right.$ de nodos totales del elemento $=3$ ), el vector que almacena las incógnitas (en forma transpuesta) será:

$\alpha_{\mathrm{m}}^{\mathrm{T}}=\left\{\mathrm{T}_{1}, \mathrm{X}_{2}, \mathrm{I} 1_{3}, \mathrm{I} 2_{4}, \mathrm{I}_{5}, \mathrm{~T}_{6}, \mathrm{X}_{7}, \mathrm{I} 1_{8}, \mathrm{I} 2_{9}, \mathrm{I}_{10}, \mathrm{~T}_{11}, \mathrm{X}_{12}, \mathrm{I} 1_{13}, \mathrm{I}_{14}, \mathrm{I}_{15}\right\}$

donde $\mathrm{T}_{1}, \mathrm{X}_{2}, \mathrm{I}_{3}, \mathrm{I}_{4}$ y $\mathrm{I}_{5}$ son las incógnitas asociadas al nodo local $\mathrm{N}^{\mathrm{o}} 1 ; \mathrm{T}_{6}, \mathrm{X}_{7}, \mathrm{I}_{8}, \mathrm{I}_{9}, \mathrm{I}_{10}$ están asociadas al nodo $\mathrm{N}^{\mathrm{o}} 2$ y por último $\mathrm{T}_{11}, \mathrm{X}_{12}, \mathrm{I}_{13}, \mathrm{I} 2_{14}, \mathrm{I}_{15}$ están asociadas al nodo $\mathrm{N}^{\circ}$ 3. Nótese que los subíndices $m$ responden a la Ec. 14.

Los términos de la matriz elemental y del vector de términos independientes son obtenidos por bucles sobre los puntos de gauss, integral sobre el elemento de las ecuaciones gobernantes que describen a cada grado de libertad. Estas integrales, obviamente, no son las mismas para todos los grados de libertad.

Los valores de $\mathrm{K}_{\mathrm{lm}}$ y $\mathrm{B}_{1}$ que describen el problema térmico ( $\mathrm{N}^{\circ}$ Dof $\left.=1\right)$ son:

$$
K_{l m}=\int \rho C_{p} \frac{\phi_{j}}{\Delta_{t}} \varphi_{i}+\int \rho C_{p} V_{z} \frac{\partial \phi_{j}}{\partial z} \varphi_{i}+\int k \frac{\partial \phi_{j}}{\partial x} \frac{\partial \varphi_{i}}{\partial x}
$$




$$
B_{l}=\sum_{j=1}^{\operatorname{NodT}}\left\{\int \rho C_{p} \frac{\phi_{j}}{\Delta_{t}} \alpha_{m}^{(n-1)} \varphi_{i}+\int g \varphi_{i}\right\}
$$

siendo:

$$
\begin{aligned}
& g=\rho\left(-\Delta H_{0}\right) \omega_{r} R_{a} \\
& \varphi_{j}=\phi_{j}+\frac{h}{2|V|}\left(V \cdot \nabla \phi_{j}\right)
\end{aligned}
$$

Donde NodT es el número total de nodos del elemento, $\Delta_{\mathrm{t}}$ es el paso de tiempo, $\mathrm{g}$ es la fuente de calor, $\phi_{j}$ es la función de forma asociada al nodo $\mathrm{j}, \varphi_{i}$ es la función de peso asociada al nodo $i, \alpha_{m}{ }^{(n-1)}$ es el valor de la temperatura en el nodo $j$ calculado en el paso de tiempo anterior, h es la dimensión del elemento proyectada sobre la dirección de $\mathrm{V}$, donde $\mathrm{V}$ es el vector de velocidades.

Los valores de $\mathrm{K}_{\mathrm{lm}}$ y $\mathrm{B}_{1}$ que describen la conversión $\left(\mathrm{N}^{\circ}\right.$ Dof $\left.=2\right)$ son:

$$
\begin{aligned}
& \mathrm{K}_{\mathrm{lm}}=\int \frac{\phi_{j}}{\Delta_{t}} \varphi_{i}+\int V_{z} \frac{\partial \phi_{j}}{\partial z} \varphi_{i} \\
& B_{l}=\sum_{j=1}^{\operatorname{NodT}}\left\{\int \frac{\phi_{j}}{\Delta_{t}} \alpha_{m}^{(n-1)} \varphi_{i}+\int R_{a} \varphi_{i}\right\}
\end{aligned}
$$

Donde $\alpha_{\mathrm{m}}^{(\mathrm{n}-1)}$ es el valor de la conversión en el nodo $j$ calculado en el paso de tiempo anterior.

Los valores de $\mathrm{K}_{\mathrm{lm}}$ y $\mathrm{B}_{1}$ que describen las concentraciones de los iniciadores ( $\mathrm{N}^{\circ}$ Dof $=3,4$ o 5) son:

$$
\begin{aligned}
& \mathrm{K}_{\mathrm{lm}}=\int \frac{\phi_{j}}{\Delta_{t}} \varphi_{i}+\int V_{z} \frac{\partial \phi_{j}}{\partial z} \varphi_{i} \\
& B_{l}=\sum_{j=1}^{\operatorname{NodT}}\left\{\int \frac{\phi_{j}}{\Delta_{t}} \alpha_{m}^{(n-1)} \varphi_{i}+\int R_{I n} \varphi_{i}\right\}
\end{aligned}
$$

Donde $\alpha_{\mathrm{m}}^{(\mathrm{n}-1)}$ es el valor la concentración del iniciador en el nodo $j$ calculado en el paso de tiempo anterior y $R_{\mathrm{In}}$ es la velocidad de consumo del iniciador $\mathrm{N}^{\circ} \mathrm{n}(\mathrm{dIn} / \mathrm{dt})$.

Los términos Ra y $\mathrm{R}_{\mathrm{In}}$ son funciones complejas de la temperatura, la conversión y las concentraciones de los iniciadores (ver Ecs. 7 y 8 ) y se recalculan, dentro del mismo paso de tiempo, con las soluciones de las variables en la iteración anterior. De esta manera el sistema alcanza la solución dentro de un paso de tiempo haciendo una iteración no-lineal hasta alcanzar un error mínimo determinado por el usuario del pro- grama. Una vez alcanzada la solución dentro de un paso de tiempo, la solución del paso de tiempo actual pasa a ser la solución del paso de tiempo anterior y se repite el proceso iterativo antes descrito hasta finalizar el tiempo de cálculo.

Para el caso de la resina epoxi se trabaja con tres grados de libertad menos, porque la reacción química no necesita iniciadores para comenzar. Por lo tanto las Ecs. 22 y 23 no se tienen en cuenta.

\section{Geometría y Malla Utilizada}

Se modeló un perfil rectangular de dimensiones $0.375 \times 0.625$ in para un compuesto de resina poliéster y un $63 \%$ en peso de fibra de vidrio $(\mathrm{Wg}=0.63)$. La velocidad de tirado en este caso es de $0.00508 \mathrm{~m} / \mathrm{s}$. En este trabajo se utilizó un mallador de triángulos equiláteros del tipo "frente de generación" $\$, 9$, con refinamiento adaptativo, partiendo de una malla inicial de triángulos rectángulos mostrada en la Fig. 2a.

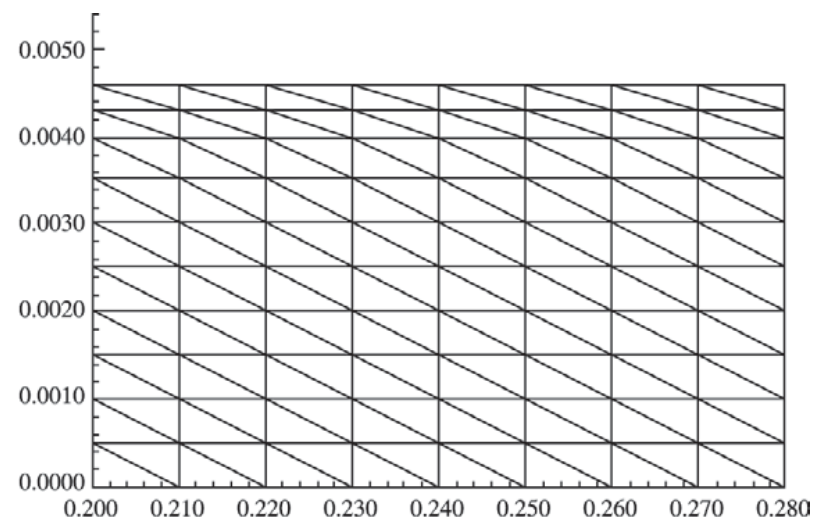

a)

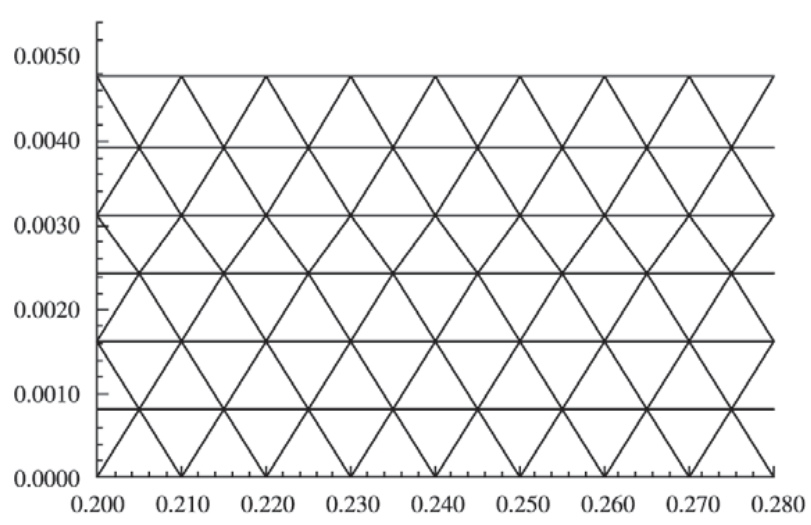

b)

Figura 2. Mallas utilizadas: a) Triángulos rectángulos b) Triángulos equiláteros. 
Se desarrolló un refinador automático de mallas dado que durante el proceso de pultrusión, para el caso de algunas resinas y para ciertas geometrías, se producen reacciones muy rápidas localizadas en zonas de pequeño tamaño, involucrando cambios de gran magnitud en las variables, con los consiguientes fuertes gradientes. Para que estos cambios puedan ser modelados con exactitud es necesario implementar un criterio para determinar el tamaño de elemento en función de la variables o sus derivadas, densificando localmente

Dado que la implementación se realizo con elementos lineales, el criterio de densificación adoptado fue la de minimizar los errores locales de interpolación. Como indicador del error local se utilizó la derivada segunda en cada nodo ${ }^{10}$. La derivada segunda se recuperó interpolando por cuadrados mínimos un paraboloide con las variables de los nodos vecinos.

El mallador genera una malla de triángulos casi equiláteros (equiláteros cuando es posible), como se mencionó anteriormente, a través de la técnica de "Frente de Generación". Inicialmente el frente de generación coincide con el contorno de la geometría que se define como uno o varios polígonos cerrados con una orientación determinada. El tamaño del Elemento a generar se determina en función de valores asignados previamente a los nodos en la grilla existente con el procedimiento antes descrito.

En la Fig. 3a se muestra la malla utilizada inicialmente para resolver el caso del compuesto poliester insaturado - fibra de vidrio, para una velocidad de tirado de $0.01016 \mathrm{~m} / \mathrm{s}$. A partir de la solución obtenida de esta primera simulación, se aplicó el refinador de mallas descripto anteriormente, obteniéndose la malla que se muestra en la Fig. 3b. Con una siguiente aplicación del refinador se obtuvo la malla de la Fig. 3c, para la cual los resultados obtenidos, no varían sustancialmente respecto a los obtenidos con la malla anterior.

\section{Resultados y Discusión}

En la Fig. 4 se pueden ver los datos experimentales de Sumerak $^{3}$ de la temperatura en el centro del perfil en función de la posición en el molde para el caso de un compuesto de resina poliéster y un $63 \%$ en peso de fibra de vidrio ( $\mathrm{Wg}=0,63)$. Se representa además la temperatura simulada en el centro de un perfil rectangular de dimensiones $0,375 \times 0.625$ in para tres velocidades de tirado diferentes $($ VEL1 $=0,00508 \mathrm{~m} / \mathrm{s} ;$ VEL2 $=0,01016 \mathrm{~m} / \mathrm{s}$; VEL3 $=0,01524 \mathrm{~m} / \mathrm{s}$ ). Las líneas continuas representan las simulaciones y los puntos las mediciones experimentales.

De la misma manera que lo mostraron Moschiar et al. ${ }^{1}$ resolviendo el sistema en diferencias finitas, este modelo numérico también predice satisfactoriamente el perfil de temperaturas, donde las temperaturas de los picos y su ubicación en el molde están dentro del error experimental.

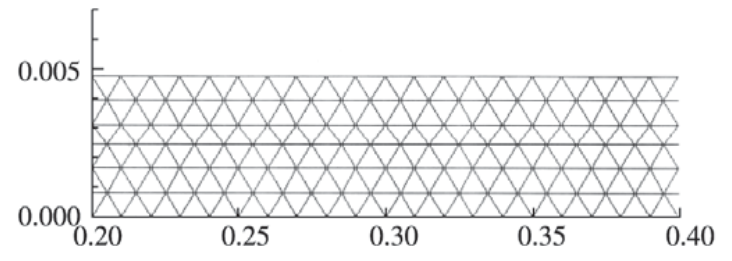

a)

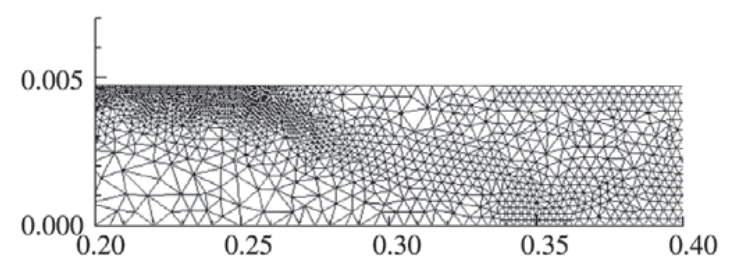

b)

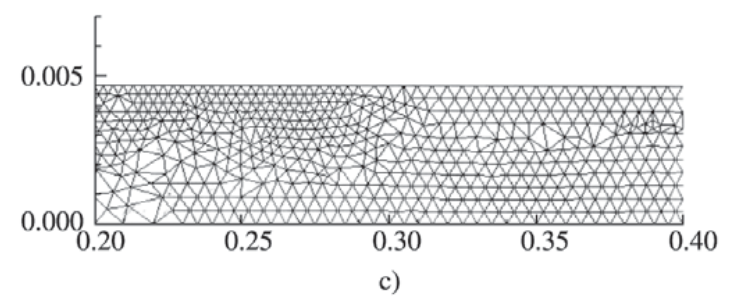

Figura 3. a): Primer refinamiento ( 353 nodos y 594 elementos); b) Segundo refinamiento (1151 nodos y 2158 elementos); c) Tercer refinamiento (3986 nodos y 7738 elementos).

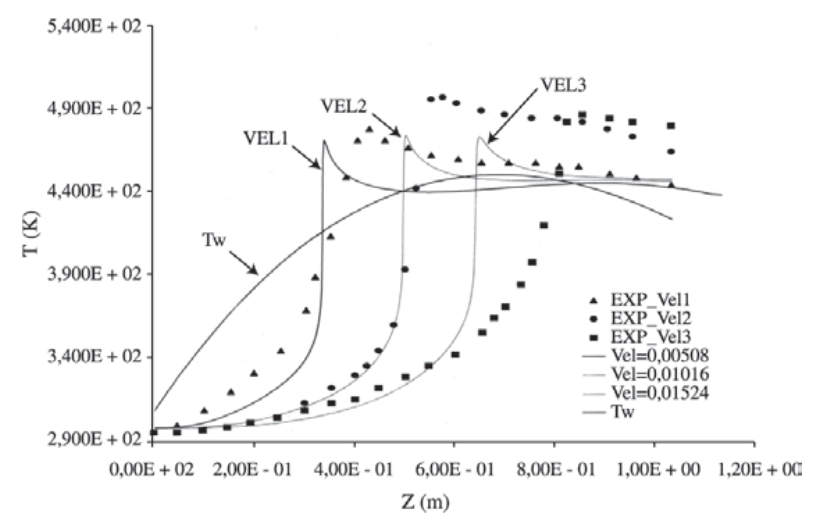

Figura 4. Perfil de temperatura a lo largo del molde para una resina poliéster.

En las Figs. 5 y 6 se representan la temperatura experimental de Hunter ${ }^{4}$ del centro de una barra circular de $\varnothing=12,7 \mathrm{~mm}$ para un compuesto de matriz epoxi con $85 \%$ de fibras de vidrio en peso, junto con la variación de la temperatura simulada por el modelo del centro de la barra en función de la posición del molde para dos perfiles de temperatura de pared diferentes $(\mathrm{Tw}=\mathrm{F}(\mathrm{I})$ y Tw $=\mathrm{F}$ (II) representa- 


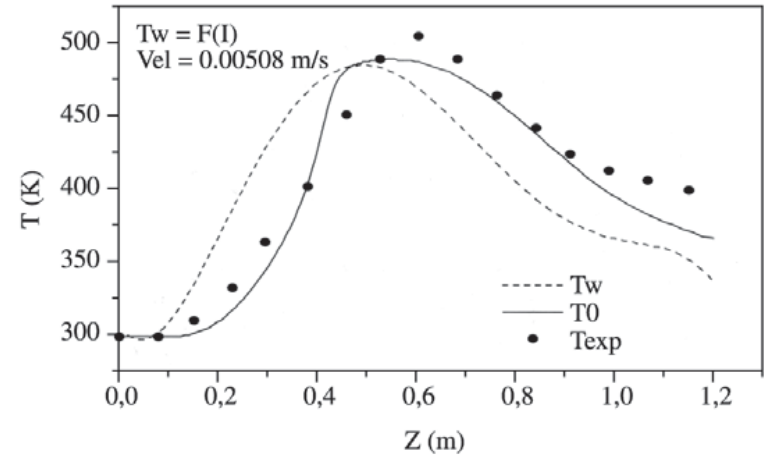

Figura 5. Perfil de temperatura para una resina epoxi para $\mathrm{Tw}=\mathrm{F}(\mathrm{I})$

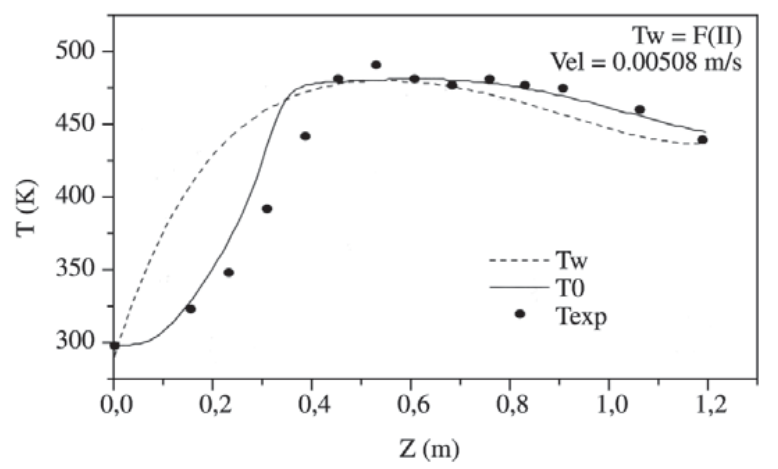

Figura 6. Perfil de temperatura para una resina epoxi para $\mathrm{Tw}=\mathrm{F}(\mathrm{II})$.

dos en la misma figura) y una velocidad de tirado $\mathrm{Vz}=0,00508 \mathrm{~m} / \mathrm{s}$.

La correspondencia entre el modelado numérico y los datos experimentales es también buena como en el caso del compuesto de poliéster. En este caso las predicciones se ajustan mejor que las obtenidas por diferencias finitas alcanzando un pico de temperatura máxima algo menor que el obtenido experimentalmente, pero dentro del error del experimento.

Los resultados satisfactorios obtenidos permiten utilizar el modelo numérico-computacional aquí desarrollado en la amplia gama de casos que surgen a partir de la variación de los parámetros más relevantes como las distintas composiciones, velocidades de tirado y geometrías. Debiendo destacarse la versatilidad del modelo para afrontar económicamente dichas variaciones lo cual posibilitará el uso de los datos obtenidos, en modelos mecánicos con vistas a la predicción de las tensiones residuales térmicas de las piezas, de las presiones internas y de las fuerzas de tirado que son función del perfil de temperaturas obtenido, para distintas geometrías y distintos compuestos.

\section{Conclusiones}

Se implementó la resolución numérica por el método de Elementos Finitos del modelo de transferencia de calor en un proceso de pultrusión para dos compuestos diferentes. Los resultados obtenidos están en buen acuerdo con los datos experimentales, lo que permitirá utilizarlos para la predicción de propiedades termomecánicas. El modelo se presenta como robusto en la medida que es aplicable a situaciones bastante disímiles desde el punto de vista de las geometrías y los materiales involucrados, presentando además gran versatilidad para la implementación de los diferentes casos, lo que facilita la implementación de la gran cantidad de casos que se derivan debido a las posibles variaciones de los parámetros más relevantes. Por otra parte, como era de esperar, los resultados obtenidos son congruentes con modelos anteriores realizados con la técnica de Diferencias Finitas, debiendo destacarse las considerables ventajas que el nuevo modelo - implementado a través de la Técnica de Elementos Finitos - conlleva en cuanto a la fácil implementación de las variaciones geométricas, condiciones de borde y densificaciones automáticas localizadas, características todas que le confieren al presente modelo indudables ventajas desde el punto de vista de la calidad y sencillez de implementación como así también respecto de la economía de cálculo.

\section{Referencias}

1. Moschiar S.M.; Reboredo M.M.; Kenny J.M. and Vazquez A. Analysis of pultrusion processing of unsaturated polyester resin with glass fibers, Polymer Composites, v. 17, n. 3, p. 478, 1996.

2. Moschiar, S.M.; Reboredo, M.M.; Larrondo, H.; Vazquez, A. "Pultrusion of epoxy matrix composites. Pulling force model and thermal stress analysis”, Polymer Composites, v. 17, n. 6, 1996.

3. Sumerak, J.E. "Pultrusion die design optimisation opportunities using thermal finite element analysis techniques”, 19th Anual Conference, Composite Institute. The Society of the Plastics Industry, Inc., Feb, 1994.

4. Joshi, S.C.; Lam, Y.C. "Three-dimensional finite-element/nodalcontrol-volume similation of the pultrusion process with temperature-dependent material properties including resin shrinkage", Composites Science and Technology, v. 61, p. 1539-1547, 2001.

5. Urquiza, S. et al. An application framework architecture for FEM..., Mec. Comput. VXXI, p. 3099-3109, 2002.

6. Sumerak, J.E. Revista de Plásticos Modernos, p. 356, 1986.

7. Hunter, G.A. 43rd Anual Conf. Composites Institute, The Society of Plastics Industry, February 1986.

8. Geroge, P.L.; Seveno, E. "The advancing-front mesh generation method revisated". International Jounal Num. Meth. In Engeneering, v. 37, p. 3605-3619, 1994.

9. Dari, E.; Venere, M.; Feijoo, R. "Finite element 3-Dmesh generation using the advancing Front technic", Mecánica Computacional, S. Idhelson,V.Sonzogni ed., AMCA, Sta Fé, Argentina, v. 14, p. 512-519,1994.

10. Zienkiewicz, O.C.; Taylor, R.L. The finite element method, McGraw Hill, Vol. III "Fluid Dynamics”, 5ta Ed., 1999. 